\title{
Polarization Properties of Intraday Variable Blazars
}

\author{
A. Kraus, T. P. Krichbaum, \& A. Witzel \\ Max-Planck-Institut für Radioastronomie, 53010 Bonn, Germany
}

\begin{abstract}
Intraday variability (IDV) is a common phenomenon within blazars, and is seen in about $30 \%$ of all sources in this class. Here we report on IDV observations carried out with the $100 \mathrm{~m}$ telescope of the MPIfR, especially focusing on variations of the linear polarization.
\end{abstract}

\section{Observations}

After the discovery of rapid flux density variations in the radio regime (Witzel et al. 1986, Heeschen et al. 1987), it turned out that IDV is seen in a significant portion of compact flat-spectrum radio sources. Statistical investigations showed that IDV occurs only in sources which are compact on VLBI (mas-) scales, but no dependence on either the optical identification, the redshift, or the galactic latitude was found (Quirrenbach et al. 1992). Observations with the $100 \mathrm{~m}$ telescope of the MPIfR revealed variability not only in the total intensity, but also in the linear polarization (polarization degree and position angle).

Using a polarization calibration method, based on the derivation of a correction matrix (Turlo et al. 1985), and frequent observations of polarization calibrator sources, we achieved an accuracy of about $5 \%$ for the individual measurement of the polarized flux density and $\leq 5^{\circ}$ for the polarization angle. The rms for highly polarized (i.e. pol.deg. $\geq 5 \%$ ), non-variable sources is $\leq 2 \%$ and $\leq 1^{\circ}$, respectively.

So far, 48 blazars of the northern hemisphere were investigated for IDV; in 17 sources this phenomenon occurred at least once. Out of these we have analyzed polarization data for 8 sources, all of them showing polarization-IDV (see fig. 1). In some cases (e.g., 0917+624) the variations of the polarization are larger than those of the total intensity. For the polarized intensity the strength of the variability can exceed a factor of 2 ; polarization angle variations range from a few degrees up to more than $100^{\circ}$. At least for the two best observed cases, a pronounced correlation between total and polarized intensity can be seen.

\section{Interpretation}

Radio IDV is a serious challenge for existing models to explain AGN variability. The variations of $1739+522$ (fig. 1) yield via the light travel time argument a brightness temperature of $>3 \cdot 10^{18} \mathrm{~K}$, far in excess of the inverse Compton limit. Today, it is still unclear, what causes these drastic variations. Radio-optical correlations, as seen, e. g., in 0716+714 (Quirrenbach et al. 1991) argue in favor of an intrinsic explanation of IDV. However, even in this case interstellar scattering must be present as additional effect due to small source sizes $\left(R \simeq c \cdot \Delta t_{\text {obs }}\right)$. In contrast, gravitational microlensing as an explanation for IDV is implausible due to the high duty cycle and short timescales observed (e.g., Wagner \& Witzel 1995). 

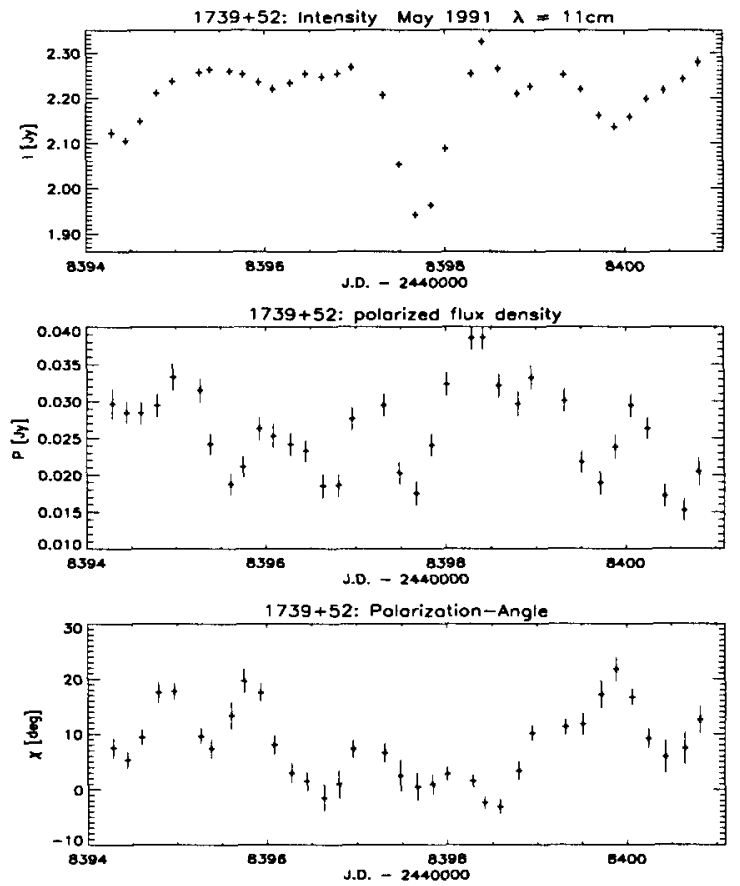

Figure 1. Rapid variability in the quasar $1739+522$ at $\lambda=11 \mathrm{~cm}$ in May 1991 . Total intensity $\mathrm{I}$, polarized intensity $\mathrm{P}$, and polarization angle $\chi$ (from top to bottom) are plotted against time. Note the rapid drop in flux density around JD 2448397.6, similar to an extreme scattering event, but much faster.

Adopting an intrinsic origin for IDV, Qian et al. 1991 considered the propagation of a thin shock through the jet plasma in a cylindrical geometry. They found this model capable of explaining the variations and the apparent high brightness temperatures by $T_{B}^{a p p}=\gamma_{s}^{2} \mathcal{D}^{3} T_{B}^{t r u e}$. As an alternative, models using collective emission processes are proposed (e.g., Benford 1992). In this framework, the origin of the emission is the scattering of relativistic electron streams in regions of plasma turbulence. Although these models do not have any difficulties to deal with high brightness temperatures, it is still unclear, whether they are working over a wide frequency range, i.e. can explain broad-band variations.

\section{References}

Benford, G. 1992. ApJ, 391, L59-62.

Heeschen, D. S., et al. 1987. $A J$, 94, 1493-1507.

Qian, S. J., et al. 1991. $A \& A, 241,15-21$.

Quirrenbach, A., et al. 1991. ApJ, 372, L71-74.

Quirrenbach, A., et al. 1992. $A \in A, 258,279-284$.

Turlo, Z, et al. 1985. $A \cup A, 142,181-188$.

Wagner, S. J., \& Witzel, A. 1995. ARA\&A, 33, 163-197.

Witzel, A., et al. 1986. Mitteilungen der Astronomischen Gesellschaft, 65, 239-241. 